\title{
Galactic Kinematics from Open Clusters $\dagger$
}

\author{
Zi Zhu \\ Department of Astronomy, Nanjing University, China \\ email: zhuzi@nju.edu.cn
}

On the basis of the astrophysical parameters of the Galactic open clusters published in the recent years, we have selected $\sim 300$ clusters completely with all measurements of their kinematical parameters. Almost all the clusters have a heliocentric distance less than $3.0 \mathrm{kpc}$. Inspecting the velocity field defined by the sample, we found some 30 clusters that have extremely large peculiar motions. All the clusters with large peculiar velocities are distant objects further than $2 \mathrm{kpc}$ from the Sun, and some of them have large Galactic height $|z|>0.35 \mathrm{kpc}$. The age ranges of the clusters are from 4 Myr to 1 Gyr with the intermediate age of 50 Myr.

The density distribution of clusters perpendicular to the Galactic plane is considered as barometric. In order to examine the relation of distribution of the clusters with their ages, we have considered two subsets of the samples: one with ages younger than $4 \mathrm{Myr}$, and another with ages elder than 4 Myr. The Galactic height defined by the younger clusters is $62 \pm 8 \mathrm{pc}$, and is $58 \pm 6$ pc for the elder clusters. Further, we found that there are several younger clusters embed in the Gould-belt that form open clusters complexes. Excluding the effect introduced by the complexes, we yielded the scale height of the open clusters $57 \pm 4$ pc.

Accepting an axisymmetric model of the Galactic rotation. we are able to derive the Oort constants $A$ and $B$ from the proper motions of clusters, while the parameter $2 A R_{0}$ can be independently obtained from the radial velocities. Using a iterative fashion, we yielded the distance to the Galactic center $R_{0}=8.03 \pm 0.70 \mathrm{kpc}$. This value is excellent coincided with the present best determination of $R_{0}$. Together with the Oort constants $A=16.16 \pm 1.07 \mathrm{~km} \mathrm{~s}^{-1} \mathrm{kpc}^{-1}$, and $B=-13.19 \pm 0.76 \mathrm{~km} \mathrm{~s}^{-1} \mathrm{kpc}^{-1}$, the present determination of $R_{0}$ suggests $V_{0}=236 \pm 23 \mathrm{~km} \mathrm{~s}^{-1}$ for the rotation speed of the Galaxy at the Sun. This value is identical with the IAU 1985 recommended value of $V_{0}=220$ $\mathrm{km} \mathrm{s}^{-1}$ within its standard deviation.

Using the tangential and radial velocities of the clusters, the rotation curve defined by the open clusters within a range of $3 \mathrm{kpc}$ near the Sun can be constructed. The schematic structure of the curve is as alike as those given by many other investigators. The average slope of the decline of the rotation curve is $-4.6 \pm 0.8 \mathrm{~km} \mathrm{~s}^{-1} \mathrm{kpc}^{-1}$, which is even steeper than those given by Oort constants $(B-A)$. Observing rotation velocities of clusters as functions of the azimuthal angle on the Galactic plane, we find that the circular speed gradually decreases in the direction of the Galactic rotation. This fact might be an evidence for the clusters moving on the oval orbits. Using a simple potential model of the Milky Way, the closed elliptical orbits and motions defined by the kinematical measurements of the clusters are constructed. We found that the orbit's eccentricity is $e=0.10 \pm 0.03$ and the angular distance of the Sun to the pericenter is $\theta_{0}=111^{\circ} .5 \pm 16^{\circ} .0$. This solution implies that the orientation of the Sun is in the location near the minor axis of an elliptical orbit. This suggestion coincides with some other investigations of the elliptical disk-potential models based on kinematical constraints.

$\dagger$ Supported by the National Natural Science Foundation of China (Grant 10333050). 